\title{
The determination of the spectral turnover in faint Compact Steep-Spectrum radio sources using the VLA at $74 \mathrm{MHz}$
}

\author{
W. Tschager ${ }^{1}$, R. T. Schilizzi ${ }^{2}, 1$, H. J. A. Röttgering ${ }^{1}$, I. A. G. Snellen ${ }^{3}$, G. K. Miley ${ }^{1}$, and R. A. Perley ${ }^{4}$ \\ ${ }^{1}$ Leiden Observatory, PO Box 9513, 2300 RA Leiden, The Netherlands \\ 2 Joint Institute for VLBI in Europe, PO Box 2, 7990 AA Dwingeloo, The Netherlands \\ ${ }^{3}$ Institute for Astronomy, Royal Observatory, Blackford Hill Edinburgh, EH9 3HJ, UK \\ ${ }^{4}$ National Radio Astronomy Observatory, PO Box O, Socorro, NM 87801, USA
}

Received 3 December 2002 / Accepted 19 February 2003

\begin{abstract}
We carried out VLA observations in A configuration at $74 \mathrm{MHz}$ of a new sample of faint Compact Steep-Spectrum (CSS) radio sources as part of a comprehensive project to study the nature and evolution of peaked-spectrum radio sources. In conjunction with higher frequency spectral information this has enabled us to determine the peak frequency and peak flux density of the spectral turnover for 35 faint CSS radio sources. Spectra are presented. An analysis is given of the quality of the images and their suitability for integrated intensity measurements of the CSS radio sources.
\end{abstract}

Key words. radio continuum: galaxies - galaxies: active - galaxies: evolution

\section{Introduction}

Gigahertz-Peaked Spectrum (GPS) sources and Compact Steep-Spectrum (CSS) sources are characterised by a convex radio spectrum. GPS radio sources have spectra which peak at a frequency of about $1 \mathrm{GHz}$ and have sizes of typically 10 to $100 \mathrm{pc}$. CSS radio sources have spectra which peak around $100 \mathrm{MHz}$ and have sizes of typically a few to a few tens of kpc.

The youthfulness of GPS radio galaxies has been established beyound reasonable doubt. The measurements of the outward motion of hotspots found for several GPS radio galaxies provide the best direct evidence for their very young age (e.g. Owsianik \& Conway 1998; Tschager et al. 2000). Hotspot advance speeds have been found typically in the range from 0.1 to $0.2 h^{-1} c$, translating into kinematic ages of a few hundred to a few thousand years. For CSS radio galaxies, radiative ages have been deduced within the framework of synchrotron and continuous injection theory from the highfrequency ageing break by Murgia et al. (2002). They find ages of about $10^{5}$ years, supporting the evolution scenario in which GPS sources grow larger to become CSS sources.

However, since it is not yet possible to measure directly the outward motion of CSS hotspots, other means are necessary to establish a clear evolutionary link between GPS and CSS sources. Snellen et al. (2000) investigated observational characteristics common to both classes of objects. Correlations were found to exist between their peak flux density, peak frequency and overall angular size. These correlations reveal an underlying evolutionary link. Assuming the dominating

Send offprint requests to: I. A. G. Snellen, e-mail: ignas@roe.ac.uk physical process responsible for the spectral peak is synchrotron self absorption (SSA), these correlations demonstrate that the ratio between the overall angular size and the angular size of the dominant radio components producing the peaked spectrum is constant for the different samples of bright and faint GPS and bright CSS sources. This suggests that peaked spectrum sources evolve in a self-similar way. Thus the acronyms GPS and CSS appear not only to describe different classes of peaked spectrum objects, but also to label different evolutionary stages in the life of individual radio sources.

These conclusions are not correct if free-free absorption (FFA) is the dominating physical process responsible for the low frequency turnover. FFA originates when the radio emission passes through a foreground screen of ionised plasma, while SSA is internal to the radio source. Attempts to determine which process dominates the low frequency absorption of the radiation have been inconclusive. While FFA has been found to be important in some GPS radio galaxies that are highly polarised, are variable, or have a highly inverted low frequency spectrum (Tingay et al. 2003; Inoue et al. 2003), most of them do not require this alternative process to explain their convex spectral shape. It is unlikely that any external envelope of ionised plasma screening the radio emitting region is as large as a CSS source, so the dominance of FFA over SSA decreases and becomes unimportant with ongoing source growth.

Apart from the evolution in size, radio galaxies of all ages are expected to undergo an evolution in luminosity. Classical double radio galaxies which are no longer engulfed by their optical host galaxy decrease their total radio luminosity while growing larger. This is due to adiabatic losses the relativistic 
plasma suffers during its pressure driven expansion out of the hotspot region back into the lobe environement (e.g. Blundell et al. 1999; Kaiser \& Alexander 1997, 1999; Kaiser et al. 1997). In the final stages in the life of radio galaxies, the inverse Compton scattering process continues to sustain the radiative losses even longer.

Snellen et al. (2000) suggest that these late phases of radio source evolution are preceeded by a period of increase in radio luminosity which lasts as long as the radio galaxy is confined within the core radius of the host galaxy. Such an evolution scenario is expected for a ram-pressure confined radio source in a surrounding medium with a King profile density. In the inner parts of the King profile, the density of the medium is roughly constant allowing the radio source to build up its luminosity. After it has grown large enough the density of the surrounding medium declines and the luminosity of the radio source decreases. A comparison of the local luminosity function of GPS galaxies with that of extended sources is a good test for this evolution scenario, but still needs observational confirmation (Snellen et al. 2003).

Until recently, detailed population studies of young radio sources have been limited to samples of bright GPS sources (Stanghellini et al. 1998), bright CSS sources (Fanti et al. 1990) and faint GPS sources (Snellen et al. 2000). A review of the properties of these samples can be found in O'Dea (1998). To complement these studies we embarked on a project to investigate a new sample of faint CSS radio sources. This paper describes VLA A-array observations at $74 \mathrm{MHz}$ carried out on this sample. The goal of these observations was to determine the peak frequency and peak flux density of these CSS sources. This is crucial for investigating how the members of our new faint sample relate to their bright relatives and to the bright and faint GPS radio galaxies. This will allow modelling the growth process of peaked-spectrum radio sources at early stages of their life over a wide range of physical size and intrinsic radio luminosity, thereby reproducing the self-similarity characeristics of the growth process.

The sample of CSS radio sources under investigation is described in Sect. 2. Section 3 deals with the data reduction, quality control and source extraction. In Sect. 4 we use the new data to determine the frequencies and flux densities of the spectral turnovers and discuss the results in Sect. 5.

A number of important properties of the sources in the sample - identifications and the redshift and linear size distributions - are not discussed in this paper since they require additional radio imaging and optical imaging and spectroscopic data, to be presented in a second paper.

\section{The new sample of faint CSS radio sources}

Using the WENSS, NVSS and FIRST ${ }^{1}$ radio surveys, we have constructed a new sample of faint CSS sources to fill in an unexplored segment of the peak-flux-density - peakfrequency parameter space. Unresolved radio sources located in the area $08^{\mathrm{h}}<\mathrm{RA}<17^{\mathrm{h}}$ and $30^{\circ}<$ Dec $<43^{\circ}$ with a flux

\footnotetext{
${ }^{1}$ We used catalogue versions available in early 1998
}

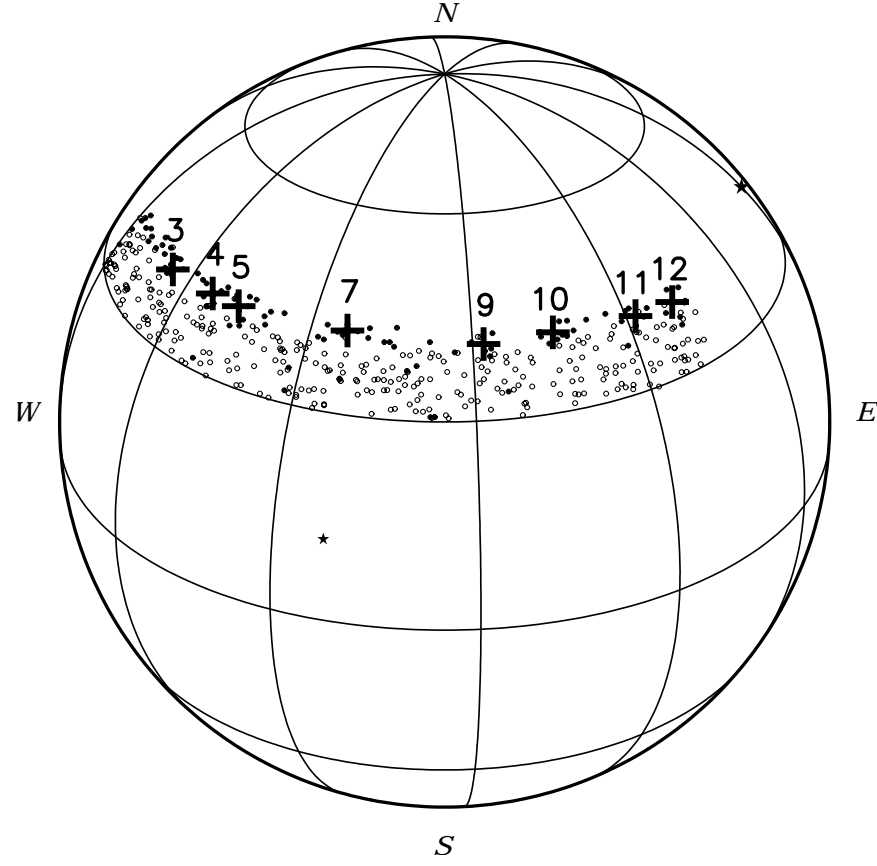

Fig. 1. Distribution of the 402 faint CSS radio sources (circles) on the celestial sphere. Graphic markers for sources belonging to the working sample (99 objects) are filled. The area of 0.429 sr searched for CSS radio sources spans from $08^{\mathrm{h}}<\mathrm{RA}<17^{\mathrm{h}}$ and $30^{\circ}<\mathrm{Dec}<43^{\circ}$. The parallels shown are - from top to bottom - drawn at Dec $+60^{\circ}$, $+30^{\circ}$, equator, $-30^{\circ}$. The spacing between the meridians is $2^{\mathrm{h}}$. The size of the + signs marking the field centres give an indication of the sensitivity area of the observations. The calibrator sources Cygnus A (big star) and Virgo A (small star) are marked.

density $>250 \mathrm{mJy}$ were selected from WENSS at $325 \mathrm{MHz}$, and their positions were correlated with the NVSS catalogue at $1.4 \mathrm{GHz}$. Sources with an optically thin radio spectrum of $\alpha<-0.5\left(S \propto v^{\alpha}\right)$ were subsequently selected. We then correlated this sample of steep-spectrum radio sources with the $1.4 \mathrm{GHz}$ FIRST catalogue to select only those sources with a deconvolved size of $<2$ ". This selection on spectral index and angular extension is the same as used by Fanti et al. (1990) for selecting the reference sample of bright CSS sources mainly from the 3CR catalogue. From a total of 402 objects found, we selected a working sample composed of 99 sources, mostly located in the region of the sky limited by $08^{\mathrm{h}}<\mathrm{RA}<17^{\mathrm{h}}$ and $37^{\circ}<$ Dec $<43^{\circ}$ (see Fig. 1).

In order to obtain an uniform distribution over as wide a range of spectral indices as possible, some of the steepest spectrum objects were taken from outside this area and added to the list in such a way that the final working sample has an almost uniform spectral index distribution in the range $-0.5<\alpha<-1.2$. This sample now contains enough sources to allow a division of objects into redshift, peak frequency, peak flux density and spectral index bins, each containing statistically significant numbers (see Fig. 2, left panel). The histogram shown in the right panel of Fig. 2 shows the normalised integrated distribution of flux density at the selection frequency of $325 \mathrm{MHz}$ and illustrates the faintness of the sample. 

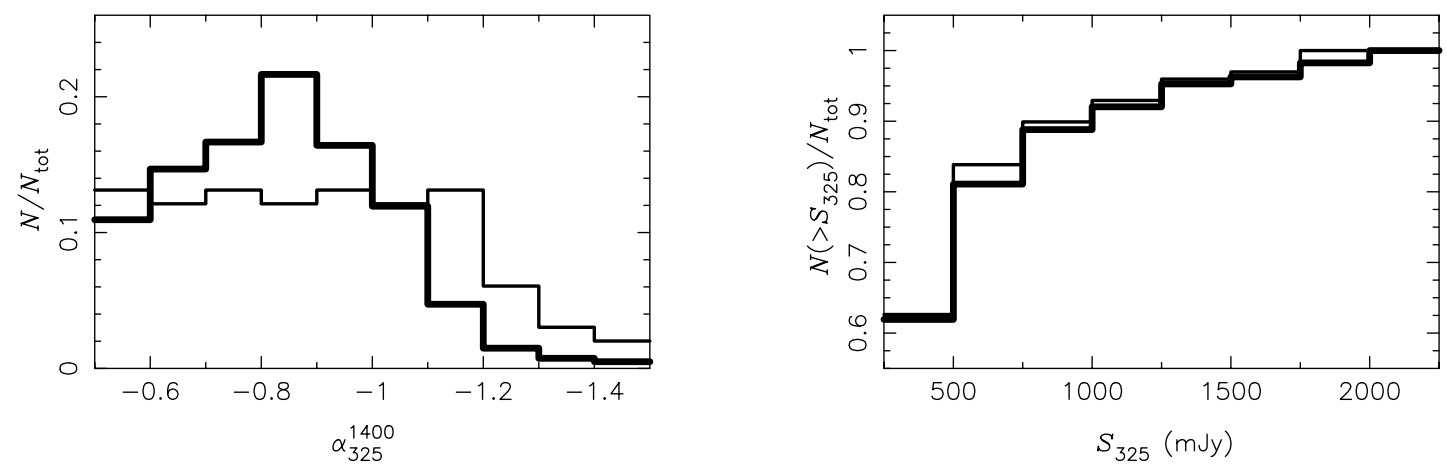

Fig. 2. Left panel: normalised distribution of spectral index $\alpha$ between $325 \mathrm{MHz}$ (WENSS) and $1.4 \mathrm{GHz}$ (NVSS) of the whole sample (thick line) and the working sample (thin line). Right panel: normalised "integrated" distribution of flux density at the selection frequency of $325 \mathrm{MHz}$ (WENSS) of the whole sample (thick line) and the working sample (thin line). More than $60 \%$ of the objects are fainter than $500 \mathrm{mJy}$ at this frequency. The last bin contains objects with $S_{325}>2000 \mathrm{mJy}$. The whole and working sample contain 402 and 99 sources, respectively.

\section{74-MHz observations with the VLA in A configuration}

\subsection{The observations}

The observing mode chosen had $6.6 \mathrm{~s}$ integration time, 1.6 MHz total bandwidth, 127 channels and 2 polarisations and works reliably at the highest possible dump rates of the VLA correlator. This mode is used to facilitate Radio Frequency Interference (RFI) rejection and to keep bandwidth smearing and time averaging effects at tolerable limits.

Test runs conducted at the VLA had shown that $50 \mathrm{mJy} /$ beam rms-noise can be achieved outside the galactic plane with $2 \mathrm{hr}$ integration time. We estimated that the vast majority of our CSS targets have total flux densities at $74 \mathrm{MHz}$ well above $250 \mathrm{mJy}$. Thus, our set-up allowed detections at $>5 \sigma$ for targets with correlated flux density $>250 \mathrm{mJy}$ lying close to the pointing centres. Moving away from the pointing centres, the SNR of a detection decreases due to the loss in sensitivity and uncorrected smearing effects introduced by the ionosphere.

Since we had been granted a total of 16 hours of observating time we needed to choose a set of eight pointing centres to optimize the number of detectable sources. Figure 1 shows the distribution of the eight pointing centres chosen from the initial twelve fields proposed. The fields observed are numbered as field 3, 4, 5, 7, 9, 10, 11 and 12 from West to East. While the primary beam has a $F W H M$ of $11.7^{\circ}$, the sensitivity area around each field centre is limited to a region of $6^{\circ}$ in diameter (see Sect. 3.4).

The observations were carried out on December 26 and 29, 2000.

\subsection{Reduction of the meter-wavelength data}

The complete data reduction - calibration and imaging - was carried out with the NRAO Astronomical Imaging Processing System (AIPS).

After the initial data check the bandpasses were calibrated. Even if the inspection of the cross-power spectra showed strong, mostly internally generated, interference, the use of strong bandpass calibrators such as Cygnus A $\left(S_{\text {tot }}^{74} \sim 17 \mathrm{kJy}\right)$ or Virgo A $\left(S_{\text {tot }}^{74} \sim 1.5 \mathrm{kJy}\right)$ allow the measuring of the filter bandpasses without any pre-flagging.

In the next step the antenna gains were set and a first, coarse phase calibration was performed. Again, because of its strength Cygnus A was by far the best choice of calibrator. For the actual calibration run, an RFI-spike-free region of the spectrum was used. These gain solutions, consisting of a single phase and amplitude solution, were applied to the data.

The data reduction process entails a careful removal of RFI. Typically some $15 \%$ of the data were flagged. Following flagging, spectral averaging reduced the volume of data. Allowing for $5 \%$ amplitude reduction at a distance of $3^{\circ}$ from the pointing centre, we were able to reduce the total amount of data by a factor of eight.

We followed the traditional self-calibration strategy (Perley 2001) which is based on using background objects lying within a certain distance from the pointing centre as a model for phase calibration. The best choice would be to use an area smaller than the isoplanatic patch. However, because the size of isoplanatic patch is essentially unknown we used objects within $3^{\circ}$ from the pointing centre. WENSS provided models for all our fields. Each background source was modeled by a $\delta$-component and scaled by its estimated total flux density. The amplitude of the $\delta$-component is estimated by extrapolation from its WENSS flux density assuming a constant spectral index of -0.8 . In general, the phase solutions are quite insensitive to minor variations in spectral index between 325 and $74 \mathrm{MHz}$. Phase errors can result when some of the WENSS objects are invisible at $74 \mathrm{MHz}$. However, we found that adopting this method for phase self-calibrating our data works very robustly for models containing a total flux density of at least $50 \mathrm{Jy}$.

The noise level in our images is set by the sidelobes of confusing objects in the field of view, and required that the whole primary beam of $11.7^{\circ}$ be imaged. Very bright objects outside the primary beam can also affect the image quality, and were included in the deconvolving process as well. The phase self-calibrated uv-data were deconvolved using a noncoplanar, wide-field image deconvolution algorithm which is implemented in the main AIPS imaging task, IMAGR. Two clean-selfcal cycles were performed, and the images obtained showed indeed the expected rms-noise level and a myriad of 
objects. The convolving beam was chosen to be a circular Gaussian function with FWHM 24".

\subsection{Assessing the quality of the images}

The section of the image in Fig. 3 illustrates a minor defect seen in all images. The background noise shows low-level, horizontal stripes apparently originating at the celestial North pole. They have typical amplitudes of $<1.5 \sigma$, but can rise locally up to $2.5 \sigma$. Their characteristic pattern is due to unflagged, long-lasting, low level stationary RFI (Perley 2002). It does not critically affect the image quality, or the accuracy of source extraction in the inner part $\left(r \lesssim 3^{\circ}\right)$ of the imaged area. However, we observed when moving away from the pointing centre the ripples can (locally) rise above $3 \sigma$ leading to spurious source identifications.

In addition to this site-related interference the signals to be correlated have already suffered distortion by the ionosphere when arriving at the receivers. Data processing based on simple self-calibration is unable to handle entirely correctly effects due to ionospheric disturbances, and consequently we investigated the image quality degradation it causes.

During our observations we observed the temporary effect sunrise has on the ionosphere, and how it degraded the quality of the recorded visibilities. The impact on the East- and Westarm antennas was much more severe than on the North-arm antennas. Between local sunrise and the onset of severe uncorrectable phase unstability there was a delay of about one hour. The disturbance itself persisted for about one hour. After this period the ionosphere stabilized sufficiently to allow the VLA correlator to track phase windings occuring at rates as high as $0.26 \mathrm{rad} \mathrm{s}^{-1}$. Data recorded during the temporal ionospheric instability - some $10 \%$ of the total amount - had to be removed.

Figure 4 shows phase solutions for antenna N72 for field 5 (top panel) and field 12 (bottom panel). The observations of field 5 took place before sunrise, those for field 12 after sunrise. The antenna label N72 describes the position of the antenna along the North arm of the VLA. N72 is the outermost antenna on the $\mathrm{N}$-arm. All phase measurements are referenced to Antenna N8, the innermost $\mathrm{N}$-antenna. Comparing the timedependence of the phase corrections for field 5 and field 12 on the N72-N8 baseline the effects of sunrise on the ionosphere, and consequently on the data quality are visible.

The ionosphere is unstable not only on temporal scales, but also inhomogenous on spatial scales. The isoplanatic patch is a measure of the size of spatially stable regions. The definition of isoplanatic patch depends on the context in which the concept is used. We define the isoplanatic patch as the area on the sky for which one single phase solution (per time tag) can be applied without creating effects of image distortion. The size of the isoplanatic patch when observing in A-array is a priori unknown, but smaller than the primary beam, and probably as small as $6^{\circ}$ in diameter during our two observing sessions. This assertion is based on our empirical experience gained during data processing, and is supported by the fact that individual objects more than a few degrees apart from each other were

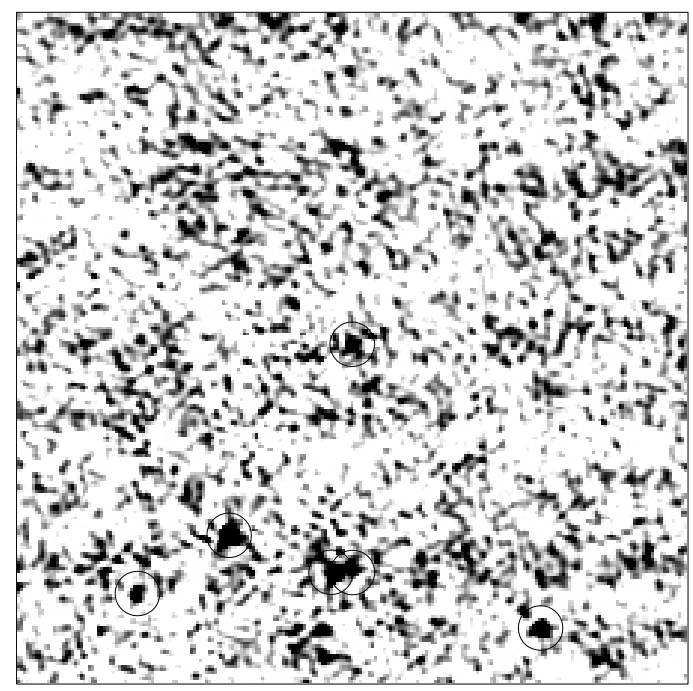

Fig. 3. Example for image degradation due to RFI affected data: $30^{\prime} \times$ $30^{\prime}$ image cutout of field 10 . Six identified field objects are circled. The rms-noise is $50 \mathrm{mJy} / \mathrm{beam}$. The colour-coding applies darkening grey shades to pixels in the range from 0 to $1.5 \sigma$.

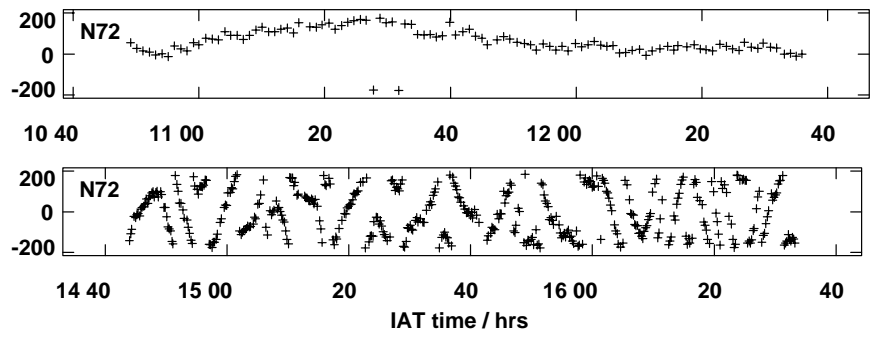

Fig. 4. Top panel: phase solutions (L-polarisation) for field 5 for antenna N72. Averaging time is $9 \times 6.6 \mathrm{~s}$. These solutions show extraordinary phase stability for a baseline of this length. Bottom panel: phase solutions (L-polarisation) for field 12 for antenna N72. Averaging time is $2 \times 6.6 \mathrm{~s}$. The phase stability shows normal behaviour.

observed to move around by several beams on timescales of tens of minutes in a completely uncorrelated fashion.

Therefore, by transfering phase corrections determined locally for an area around the pointing centre with diameter $6^{\circ}$ to regions outside this area, introduces random phase errors, and results in problems with deconvolution. In general the apparent peak intensity of an object is reduced by this effect. The integrated intensity - the quantity we wish to obtain for our target objects - remains unaffected by this smearing providing the source wandering totally lies within the integration box, and the ionospheric distortions are not so severe as to multiply image the objects.

\subsection{Source extraction}

The parameters for the sources were obtained using a Gaussian fitting procedure as implemented in the AIPS task SAD. In addition to determining the integrated intensities of the faint CSS radio sources, we extracted a list of detected objects from each field down to a cutoff level of $5 \sigma$ above the background noise. The central noise levels of the background vary slightly 
Table 1. Background noise (in mJy) determined from pixel value histogram over an area of $6^{\circ} \times 6^{\circ}$. Source density (in $\mathrm{deg}^{-2}$ ) calculated over an area of $28.3 \mathrm{deg}^{2}$ defined by $r<3^{\circ}$.

\begin{tabular}{lrrrrrrr}
\hline \hline Field & \multicolumn{1}{c}{5} & \multicolumn{1}{c}{10} & \multicolumn{1}{c}{3} & \multicolumn{1}{c}{4} & \multicolumn{1}{c}{7} & \multicolumn{1}{c}{12} & \multicolumn{1}{c}{9} \\
\hline Noise & 46.0 & 49.7 & 50.1 & 50.5 & 55.0 & 56.4 & 64.1 \\
Density & 2.4 & 3.7 & 3.7 & 1.7 & 3.3 & 2.3 & 2.3 \\
\hline
\end{tabular}

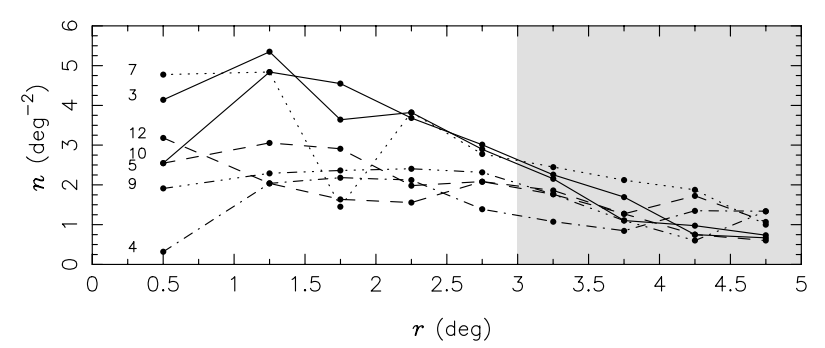

Fig. 5. Number density $n$ for sources with correlated flux density $>320 \mathrm{mJy}$ as a function of distance from the pointing centre $r$. The numbers on the left indicate the field.

between the different fields and are given in Table 1. The flux densities at $74 \mathrm{MHz}$ of the CSS target objects are given below in Table 3.

We used the source statistics to further investigate the quality of the images. Figure 5 shows source density graphs of all fields imaged for sources with correlated flux density $>320 \mathrm{mJy} /$ beam. The data for field 11 were too corrupted to allow successful imaging. Sensitivity issues due to primary beam correction and bandwidth smearing, but mostly ionospheric degradation effects are responsable for the decreasing source density with increasing radial distance $r$ observed in each field. A decline in source density indicates an increasing problem in transfering phase solutions over large distances. The discrepancies between the numbers of objects extracted from the various fields at low radial distance $r$ cannot be explained in terms of statistical fluctuations. Instead, it is very likely that most of the differences are due to actual differences in ionospheric weather conditions while the individual fields were observed. Field 4, 5,9 and 12 show relatively constant but low source densities, $n \sim 1.5-2.5 \mathrm{deg}^{-2}$. Fields 3,7 and 10 have consistently higher source densities, $n \sim 3.5 \mathrm{deg}^{-2}$, close to the pointing centre. There is no obvious correlation between background noise and source density (see Table 1). However, all fields show the onset of a clear systematic decline in source density around $3^{\circ}$ from the pointing centre.

In agreement with earlier statements about the size of the isoplanatic patch, these facts support our decision to limit the extraction area providing reliable flux-density measurements to a distance of $3^{\circ}$ from the pointing centre.

The lack of known calibrators at $74 \mathrm{MHz}$ forced us to gather circumstantial evidence to support the correctness of our integrated intensity measurements. The problem arises from the complete lack of spectral information at our observing frequency for any of the calibrators available at higher frequency. Thus, we used $4 \mathrm{C}$ objects in the fields and extrapolated the $4 \mathrm{C}-178 \mathrm{MHz}$ and higher-frequency flux densities to
Table 2. Spectral data entering the fit. Observing frequencies in GHz. Spectral points at $8.46 \mathrm{GHz}$ were obtained from a morphological study carried out with the VLA in B configuration as part of our population study of faint CSS radio sources.

\begin{tabular}{lcccccc}
\hline \hline Survey & VLA & 6C & WENSS & NVSS & GB6 & VLA \\
\hline$v_{\text {obs }}$ & 0.074 & 0.151 & 0.325 & 1.40 & 4.85 & 8.46 \\
\hline
\end{tabular}

Table 3. Peak flux densities and peak frequencies for 35 CSS sources. Flux density at $74 \mathrm{MHz}$ for $28 \mathrm{CSS}$ sources are given in Col. 2. The fitted quantities and integrated intensities have associated uncertainties of $20 \%$.

\begin{tabular}{crrrcc}
\hline \hline $\begin{array}{c}\text { Source } \\
\text { name }\end{array}$ & $\begin{array}{r}S_{74} \\
\mathrm{mJy}\end{array}$ & $\begin{array}{r}S_{\text {peak }} \\
\mathrm{mJy}\end{array}$ & $\begin{array}{c}\nu_{\text {peak }} \\
\mathrm{MHz}\end{array}$ & $\begin{array}{c}\alpha_{\text {thick }} \\
\text { (fixed) }\end{array}$ & $\alpha_{\text {thin }}$ \\
\hline $\mathrm{J} 0826+4022$ & & 385 & 227 & +0.80 & -0.84 \\
$\mathrm{~J} 0844+3953$ & & 580 & 575 & +0.80 & -2.03 \\
$\mathrm{~J} 0917+4212$ & & 452 & 521 & +0.80 & -1.39 \\
$\mathrm{~J} 0930+4033$ & 372 & 429 & 140 & +0.80 & -0.69 \\
$\mathrm{~J} 0932+3918$ & 611 & 594 & 87 & +0.80 & -0.84 \\
$\mathrm{~J} 0936+4020$ & 881 & 920 & 76 & +0.80 & -0.99 \\
$\mathrm{~J} 0938+3934$ & 494 & 500 & 105 & +0.80 & -0.64 \\
$\mathrm{~J} 1017+4130$ & 220 & 389 & 143 & +1.50 & -0.87 \\
$\mathrm{~J} 1021+3945$ & 540 & 548 & 119 & +0.80 & -1.12 \\
$\mathrm{~J} 1028+3844$ & 3730 & 3860 & 64 & +0.80 & -0.71 \\
$\mathrm{~J} 1034+4137$ & 1130 & 1062 & 126 & +0.80 & -1.19 \\
$\mathrm{~J} 1038+4229$ & 1960 & 2035 & 134 & +0.80 & -1.43 \\
$\mathrm{~J} 1049+4125$ & 1060 & 1022 & 129 & +0.80 & -0.82 \\
$\mathrm{~J} 1049+4205$ & 656 & 663 & 105 & +0.80 & -1.10 \\
$\mathrm{~J} 1055+3746$ & 2180 & 2051 & 152 & +0.80 & -1.31 \\
$\mathrm{~J} 1103+3815$ & 1190 & 1109 & 117 & +0.80 & -1.16 \\
$\mathrm{~J} 1104+3914$ & 437 & 480 & 145 & +0.80 & -1.19 \\
$\mathrm{~J} 1220+4107$ & 1120 & 1011 & 120 & +0.80 & -1.02 \\
$\mathrm{~J} 1225+4048$ & 1050 & 940 & 133 & +0.80 & -1.01 \\
$\mathrm{~J} 1244+4051$ & & 911 & 358 & +0.80 & -1.04 \\
$\mathrm{~J} 1256+4033$ & & 378 & 212 & +0.80 & -0.88 \\
$\mathrm{~J} 1408+3931$ & 842 & 872 & 102 & +0.80 & -0.93 \\
$\mathrm{~J} 1411+3846$ & 809 & 881 & 135 & +0.80 & -0.89 \\
$\mathrm{~J} 1415+4155$ & 1180 & 1154 & 107 & +0.80 & -0.81 \\
$\mathrm{~J} 1450+4121$ & 773 & 759 & 79 & +0.80 & -0.83 \\
$\mathrm{~J} 1455+3933$ & 770 & 796 & 124 & +0.80 & -1.12 \\
$\mathrm{~J} 1506+4220$ & 930 & 900 & 114 & +0.80 & -1.09 \\
$\mathrm{~J} 1513+4213$ & 385 & 402 & 142 & +0.80 & -0.95 \\
$\mathrm{~J} 1515+4042$ & 855 & 844 & 122 & +0.80 & -1.31 \\
$\mathrm{~J} 1605+3857$ & & 585 & 286 & +0.80 & -1.18 \\
$\mathrm{~J} 1644+3739$ & 262 & 497 & 148 & +1.50 & -1.12 \\
$\mathrm{~J} 1645+4010$ & 1190 & 1199 & 107 & +0.80 & -0.91 \\
$\mathrm{~J} 1645+4152$ & & 304 & 286 & +0.80 & -1.07 \\
$\mathrm{~J} 1654+3905$ & 397 & 748 & 326 & +0.71 & -0.76 \\
$\mathrm{~J} 1655+3916$ & 433 & 551 & 109 & +1.50 & -0.89 \\
\hline
\end{tabular}

$74 \mathrm{MHz}$. All 4C objects whose available radio spectral information indicates a bent spectrum have been excluded from the list of potential calibrators. Comparison of the estimated and measured integrated intensities for these calibrators allows us to claim that we do not systematically underestimate the total flux-density measurements due to source extraction and/or smearing. The scatter between the estimated and measured total flux density indicates an uncertainty in the individual 
measurements of $20 \%$, and this is also the relative error we applied to our integrated intensity measurements.

\section{Fitting the spectral turnover}

The function used by Moffet (1975) is particularly suitable for determining the extrema for peaked-spectrum radio sources. It models a peaked radio spectrum using four parameters: the spectral index below and above the turnover, $\alpha_{\text {thick }}$ and $\alpha_{\text {thin }}$, and the two peak quantities, the peak frequency $v_{\text {peak }}$ and the peak flux-density $S_{\text {peak }}$. For $\alpha_{\text {thick }}=+2.5$ the function describes the spectrum of a spherical, homogeneous, synchrotron self-absorbed radio source. The function is:

$S(v)=\frac{S_{\text {peak }}}{1-\mathrm{e}^{-1}}\left(\frac{v}{v_{\text {peak }}}\right)^{\alpha_{\text {thick }}}\left[1-\mathrm{e}^{-\left(\frac{v}{\nu_{\text {peak }}}\right)^{\alpha_{\text {thin }}-\alpha_{\text {thick }}}}\right]$.

We used Eq. (1) as the fitting function when $S_{1}-\sigma_{1} \leq S_{2}+\sigma_{2}$ was fulfilled. $S_{1}$ and $S_{2}$, and the respective errors, $\sigma_{1}$ and $\sigma_{2}$, refer to the spectral points at the two lowest frequencies $v_{1}$ and $v_{2}$, where $v_{1}<v_{2}$. This criterion expresses the tendency of the spectrum to bend at low frequency. Thirty-five objects are consistent with the criterion. Due to the low peak frequencies, the spectral turnover and the optically thick part of most CSS spectra are not well sampled by the available spectral data points. Spectral points entering the fit are given in Table 2. Measurements at $74 \mathrm{MHz}$ are available for 28 objects.

In all but one case there are insufficient data to determine or constrain the value of the optically thick spectral index. For most of our CSS objects we assumed a value of +0.8 , based on the following considerations.

De Vries et al. (1997) determined the shape of a canonical GPS spectrum using 72 strong GPS radio sources. Normalising the observed frequencies and fluxes by the source peak frequency and peak flux density allowed them to obtain a best-fit optically thick spectral index of $+0.51 \pm 0.03$. This quantity differs from the mean and standard deviation determined directly from the spectral index distribution, which is $0.57 \pm 0.30$.

Snellen et al. (2000) performed a study of 47 faint GPS radio sources. They carried out the statistical description of the spectral index distribution by taking into account the effects of the sample selection. They found that a Gaussian parental distribution with a mean of +0.80 and a standard deviation of 0.18 reproduces the observed spectral index distribution. The mean and standard deviation of the observed spectral index distribution is $0.83 \pm 0.52$, which is consistent with the corresponding value found by de Vries et al. for their sample of strong GPS radio sources. It is noteworthy that 4 objects $(8.5 \%)$ from the faint GPS sample have an optically thick spectral index steeper than +1.5 .

For objects in our own sample with $S_{1} \leq S_{2} \leq S_{3}$ and $v_{1}<$ $v_{2}<v_{3}$ the optically thick spectral index is given by the value determined from the two lowest spectral points. This information is available for one CSS object only, namely J1654+3905, with an optically thick spectral index of +0.71 . Thus, realistic values for $\alpha_{\text {thick }}$ lie in the range from +0.5 to +1.0 , and can become as inverted as +1.5 .

Attempts to fit the 35 spectra have shown that the best value for the mean sum of the reduced $\chi^{2},<\tilde{\chi}^{2}>$, is obtained by

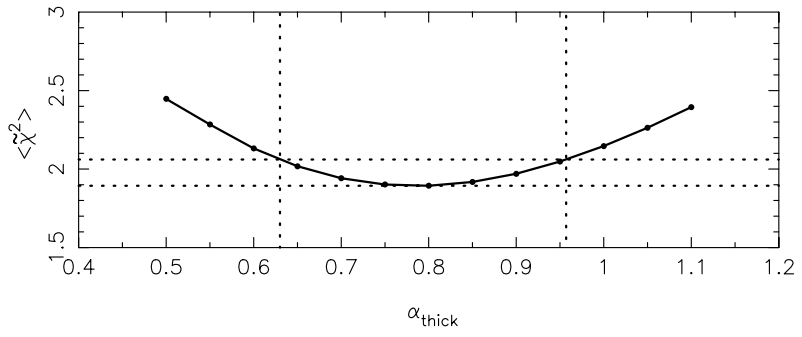

Fig. 6. The mean sum of the reduced $\chi^{2},<\tilde{\chi}^{2}>$, as function of $\alpha_{\text {thick }}$, the optically thick spectral index. The minimum of the graph occurs at +0.80 . The dotted vertical lines at +0.63 and +0.96 indicate the $68.3 \%$ confidence region for $\alpha_{\text {thick}}$.

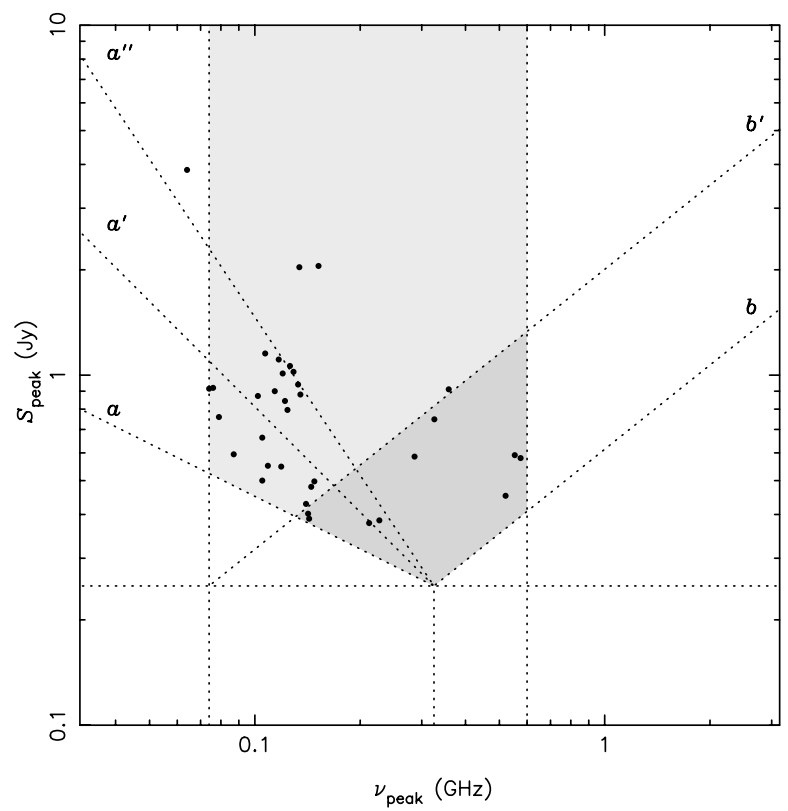

Fig. 7. Distribution of the 35 CSS radio sources on a $v_{\text {peak }}-S_{\text {peak }}$ diagram and the effect of selection criteria. The shaded area marks the region over which we expect CSS objects with $\alpha_{\text {thick }}=+0.8$ and $\alpha_{\text {thin }}<-0.5$ to be spread. The meaning of the lines $a, a^{\prime}, a^{\prime \prime}, b$ and $b^{\prime}$ is explained in the text.

taking a value of +0.8 as an estimate for $\alpha_{\text {thick }}$ in the absence of any other constraining information. The spectral points were weighted by $\left(v_{\mathrm{obs}} / \mathrm{GHz}\right)^{s}$ in order to enhance the importance of the low frequency measurements for the determination of the peak flux density and frequency, as well as to avoid divergence. On these grounds an exponent $s$ of +0.5 was chosen. Figure 6 shows the dependence of the goodness of fit on our choice for the optically thick spectral index. The stability of the fit is guaranteed by the concave shape of the graph shown. The $68.3 \%$ confidence region can be given as $0.80 \pm 0.17$, which corresponds to an uncertainty of $20 \%$. We adopt this amount for the relative uncertainty of the other two free parameters, $v_{\text {peak }}$ and $S_{\text {peak. }}$.

Three objects out of 35 show a very narrow peak and a steeper optically thick spectral index was found to improve the goodness of fit considerably. The fact that an identical percentage of sources of the faint GPS sample show similarly inverted spectrum, allows us to use a value of +1.5 instead of the overall best of +0.8 for these three sources. 

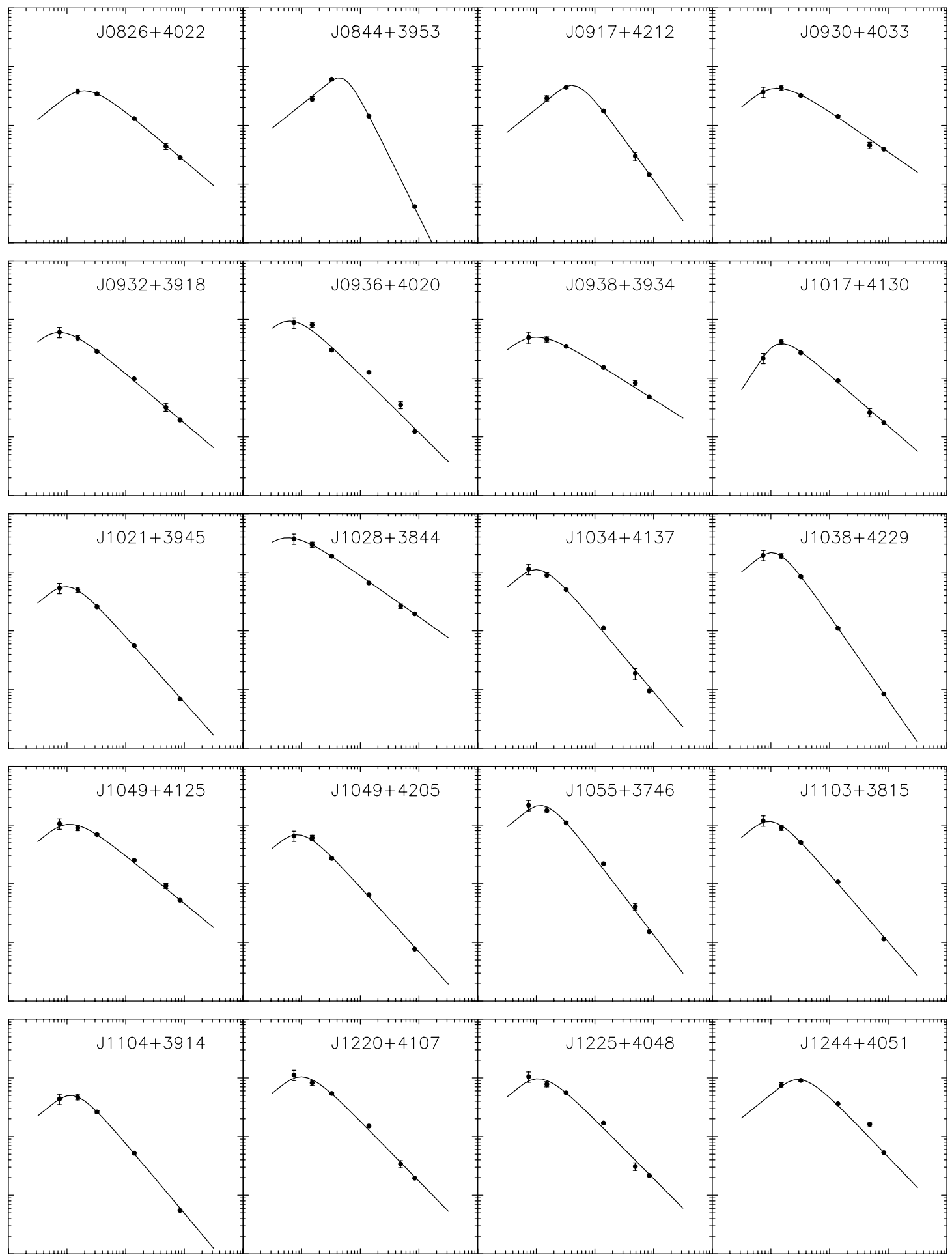

Fig. 8. Fitted spectra of CSS radio sources. The logarithmic $x$-axis covers 4 decades in frequency from $10 \mathrm{MHz}$ to $100 \mathrm{GHz}$. The logarithmic $y$-axis goes from $1 \mathrm{mJy}$ to $10 \mathrm{Jy}$. The curves show the best fits to the data. Explanation about the spectral points can be found in Table 2. Fitted parameters are listed in Table 3. More spectra in Fig. 9.

The best-fit parameters of the 35 CSS radio sources are given in Table 3.

\section{Results}

We investigated the existence of correlations between the 3 free parameters. We did not find any significant correlation between the optically thin spectral index and the peak quantities. The correlation between the two peak quantities themselves, seen in Fig. 7, can be explained in terms of selection effects.

The shaded area covers that part of the ( $\left.v_{\text {peak }}-S_{\text {peak }}\right)$ parameter space where we expect CSS objects with optically thin and optically thick spectral index of $<-0.5$ and +0.8 , respectively, 

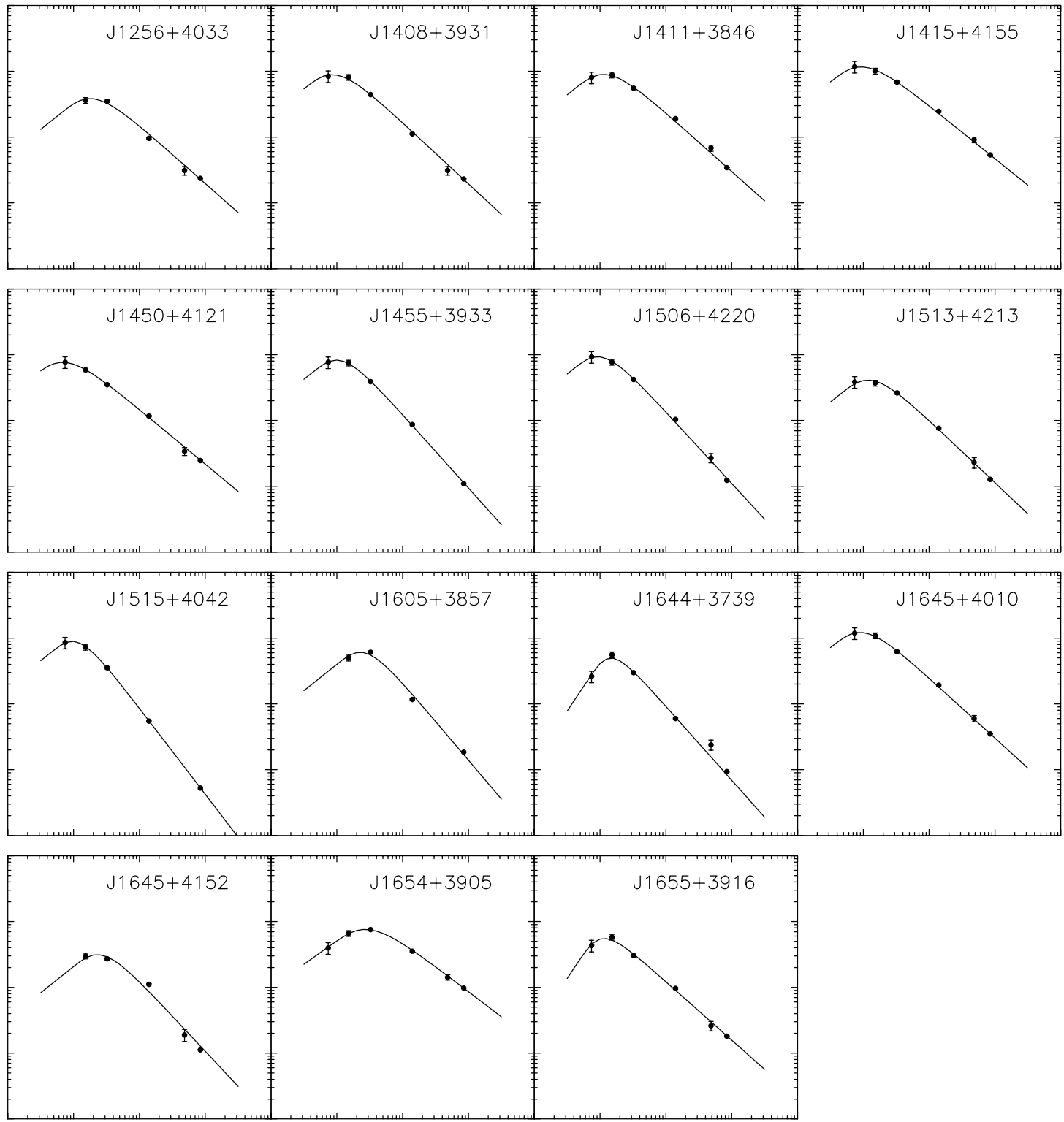

Fig. 9. Continuation of Fig. 8.

to be located. The set of dotted lines limiting the allowed part of the parameter space was calculated with the assumption of a spectrum with angled peak. Thus, small discrepancies between the subdivision into parameter space regions hosting objects with particular spectral characteristics and the location of the objects on the diagram can occur.

The dotted line $b$ has a slope of +0.8 , and marks the limit above which objects with $S_{325}>250 \mathrm{mJy}$ lie. The dark shaded area limited by the lines $b$ and $b^{\prime}$ contains objects without $74-\mathrm{MHz}$ measurements. Objects located above the dotted line $b^{\prime}$ meet the requirement $S_{74}>250 \mathrm{mJy}$.

The dotted line $a$ reflects the selection criterion $\alpha_{325}^{1400}<$ -0.5 . The artificial character of the correlation between $v_{\text {peak }}$ and $S_{\text {peak }}$ becomes evident by investigating the location on the diagram of objects with progressively steeper optically thin spectral index: objects with $\alpha_{325}^{1400}<-1.0$ need to lie above line $a^{\prime}$, objects with $\alpha_{325}^{1400}<-1.5$ above line $a^{\prime \prime}$.

Table 3 contains the results concerning the 35 faint CSS radio sources for which we were able to determine the position of the spectral turnover; Figs. 8 and 9 show the spectra.

Acknowledgements. We thank Aaron Cohen, Namir Kassim, Joe Lazio (Naval Research Lab, Washington DC, USA) and especially Eric Greisen (NRAO, Socorro, USA) for their support during the reduction of the data. WT also thanks the National Radio Astronomy Observatory (NRAO) for the hospitality offered at the AOC (Socorro, USA) during his stay in March 2001. NRAO is a facility of the National Science Foundation (NSF) operated under cooperative agreement by Associated Universities, Inc. This research has made use of the NASA/IPAC Extragalactic Database (NED) which is operated by the Jet Propulsion Laboratory, California Institute of Technology, under contract with the National Aeronautics and Space Administration. 


\section{References}

Blundell, K. M., Rawlings, S., \& Willott, C. J. 1999, AJ, 117, 677 Fanti, C., Fanti, R., Schilizzi, R. T., et al. 1990, A\&A, 231, 333

Inoue, M. 2003, PASA, 20, 1, submitted

Kaiser, C. R., \& Alexander, P. 1997, MNRAS, 286, 215

Kaiser, C. R., Dennett-Thorpe, J., \& Alexander, P. 1997, MNRAS, 292,723

Kaiser, C. R., \& Alexander, P. 1999, MNRAS, 302, 515

Moffet, A. T. 1975, in Stars and Stellar Systems, vol. IX, ed. A. Sandage, M. Sandage \& J. Kristian, p. 211

Murgia, M., Fanti, C., Fanti, R., et al. 2002, NewAR, 46, 307

O’Dea, C. P. 1998, PASP, 110, 493

Owsianik, I., \& Conway, J. E. 1998, A\&A, 337, 69
Perley, R. A. 2001, in American Astronomical Society Meeting, 199, $\# 81.01$

Perley, R. A. 2002, in EVLA Memo Series, \#49

Snellen, I. A. G., Schilizzi, R. T., Miley, G. K., et al. 2000, MNRAS, 319,445

Snellen, I. A. G., Mack, K.-H., Schilizzi, R. T., et al. 2003, PASA, 20, 1 , accepted

Stanghellini, C., O’Dea, C. P., Dallacasa, D., et al. 1998, A\&AS, 131, 303

Tingay, S. 2003, PASA, 20, 1, submitted

Tschager, W., Schilizzi, R. T., Röttgering, H. J. A., et al. 2000, A\&A, 360,887

de Vries, W. H., Barthel, P. D., \& O’Dea, C. P. 1997, A\&A, 321, 105 